Témoigner Témoigner. Entre histoire et mémoire

Getuigen Revue pluridisciplinaire de la Fondation Auschwitz

$119 \mid 2014$

Il y a 70 ans, Auschwitz. Retour sur Primo Levi

\title{
Le temps est-il sorti de ses gonds? Ascension et déclin du régime temporel de la Modernité
}

Is time out of joint? Rise and fall of the time regime of Modernity Is de tijd uit zijn voegen? Opkomst en neergang van het tijdsregime van de Moderniteit

\section{Clotilde Coueille}

\section{(2) OpenEdition}

Journals

\section{Édition électronique}

URL : http://journals.openedition.org/temoigner/1334

DOI : 10.4000/temoigner.1334

ISSN : 2506-6390

Éditeur :

Éditions du Centre d'études et de documentation Mémoire d'Auschwitz, Éditions Kimé

\section{Édition imprimée}

Date de publication : 31 décembre 2014

Pagination : 136-141

ISBN : 978-2-84174-688-0

ISSN : 2031-4183

Référence électronique

Clotilde Coueille, «Le temps est-il sorti de ses gonds ? Ascension et déclin du régime temporel de la Modernité », Témoigner. Entre histoire et mémoire [En ligne], 119 | 2014, mis en ligne le 01 janvier 2016, consulté le 23 octobre 2020. URL : http://journals.openedition.org/temoigner/1334 ; DOI : https:// doi.org/10.4000/temoigner.1334 


\section{Le temps est-il sorti de ses gonds? Ascension et déclin du régime temporel de la Modernité}

$\rightarrow$ Par Clotilde Coueille, Paris IV-Sorbonne

Aleida Assmann, Ist die Zeit aus den Fugen? Aufstieg und Fall des Zeitregimes der Moderne, Munich, Carl Hanser Verlag, 2013, 336 p.

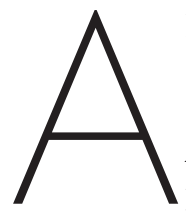

lors que ses travaux sur l'histoire et la mémoire sont désormais une référence majeure en Allemagne et ailleurs en Europe - à l'exception de la France où un seul ouvrage a été traduit - ainsi qu'aux États-Unis, Aleida Assmann entreprend, avec Ist die Zeit aus den Fugen?, une prise de recul par rapportà son objet d'étude habituel qui marque un jalon important dans sa réflexion. Auteur de plusieurs monographies sur la culture mémorielle et la construction d'une mémoire collective en Allemagne après 1945, notamment Der lange Schatten der Vergangenheit (2006) et Geschichte im Gedächtnis (2007), Aleida Assmann se propose en effet de répondre, dans cet ouvrage, à une question qui dépasse ce champ d'études : Le temps est-il « désarticulé », « déjointé »? Il s'agit tout d'abord de mettre en perspective la relation de notre société au temps, c'està-dire au présent, au passé et au futur, puis de la penser au sein d'une « culture de la mémoire » (Erinnerungskultur). Cette démarche a été initiée par des historiens - Aleida Assmann fait notamment référence aux travaux et concepts de Reinhart Koselleck et François Hartog. Pierre Nora et Christoph Cornelißen, quant à eux, ont étudié les causes de cet « avènement mondial de la mémoire » (Nora, 2002) que nous sommes en train de vivre, et qu'ils situent dans les contextes historique, social et économique des années 1970. Mais cette fois-ci, l'angle de vue est celui d'une spécialiste de la mémoire et non de l'histoire. Aleida Assmann, par la variété de ses références, ouvre la réflexion sur le phénomène mémoriel à d'autres dimensions, en l’occurrence littéraire, opérant ainsi un déplacement inédit dans le débat, à la fois transdisciplinaire et transnational.

Il n'est plus ici question simplement de l'Allemagne, mais de l'Europe, et audelà, de cet Occident qui partage un même rapport au temps apparu au moment de la Révolution française. Toute l'époque moderne en est imprégnée au point qu'il devient une caractéristique essentielle de la Modernité. Baudelaire est selon Aleida Assmann le premier à fonder la modernité sur ce nouveau rapport au temps en instaurant, comme critère de celle-ci, l'inscription de l'artiste dans le présent. Pour Baudelaire, l'artiste « cherche ce quelque chose qu'on nous permettra d'appeler la 


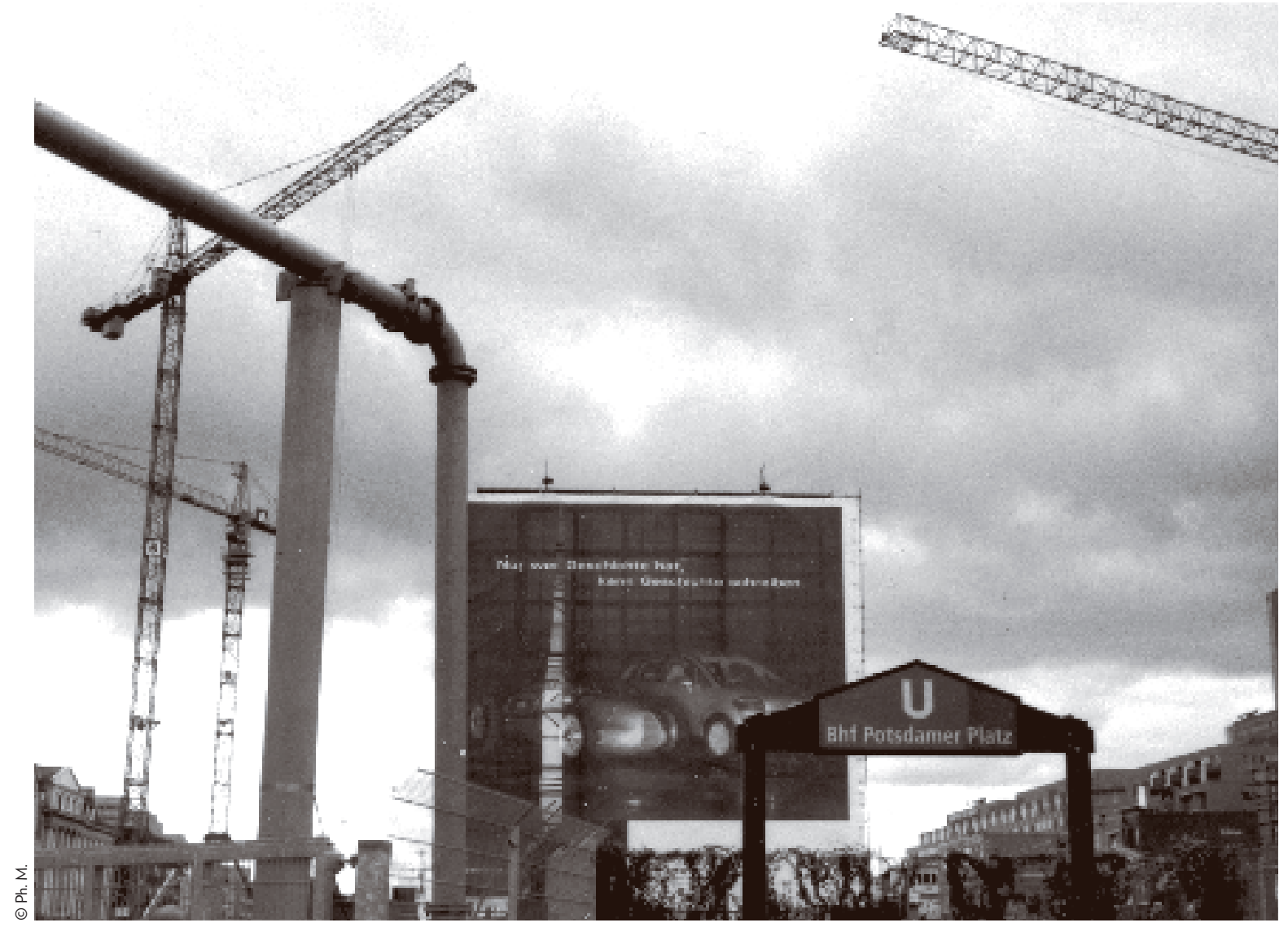

modernité. [...] La modernité, c'est le transitoire, le fugitif, le contingent, la moitié de l'art, dont l'autre moitié est l'éternel et l'immuable'. » Or si nous sommes toujours les héritiers d'une conception politique issue de la Révolution française et dans laquelle la société et ses intérêts prévalent sur les types de gouvernement, la Shoah semble d'ores et déjà avoir remplacé la Révolution dans son rôle de référence éthique ${ }^{2}$ et la « culture mémorielle » a été définie par Aleida Assmann comme résultant d'une extension de notre sentiment de responsabilité face aux crimes de masse du XX ${ }^{\mathrm{e}}$ siècle. Notre rapport au temps ne s'en trouve-t-il donc pas lui aussi modifié par ces événements ? De fait, plusieurs auteurs tels que Hans Ulrich Gumbrecht, Krzisztof Pomian ou François Furet décrivent un avenir devenu de plus en plus incertain depuis les années 1980, un temps qui serait « désorienté » (Hartog, 1995). Aleida Assmann entreprend d'étudier ce changement à l'aide du concept-outil de François Hartog de « régime d'historicité » - « régime temporel » sous sa plume - en partant de l'hypothèse de la naissance, dans les années 1980 d'un nouveau régime d'historicité qu'elle se propose de déterminer.

La volonté d'Aleida Assmann d'inscrire la recherche sur la «culture mémorielle » dans une tradition de réflexion sur le temps remontant à la fondation de l'époque moderne est reflétée par la structure tripartite du livre, qui vise à reconstituer la genèse et l'évolution du régime d'historicité de la Modernité (chapitres 1 à 3); faire
_ 1999. Panneau publicitaire pour Mercedes-Benz au cœur du chantier de la Potsdamer Platz séparée dix ans auparavant par le mur de Berlin. Sur ce panneau, on peut lire : « Seul celui qui a une histoire peut écrire l'histoire ».

(1) Charles Baudelaire, «Le peintre de la vie moderne », 1859

(2) Cf. Alon Confino, Foundational Pasts, Cambridge University Press, 2011. 
(3) Paul Ricœur, L'Histoire, la mémoire, l'oubli, Paris, Seuil, 2010

(4) Henri Bergson, L'Energie spirituelle, Paris, PUF, "Quadrige », [1919] 1985, p. 5.

(5) Shakespeare, Hamlet, acte I, scène 5 , vers 196. la lumière sur les symptômes de la crise de ce régime (chapitre 4) ; formuler enfin (chapitres 5 et 6) des postures critiques envers ce régime et des propositions pour rectifier le positionnement actuel de la recherche sur ces questions, dans une tentative de légitimation des études mémorielles aux côtés de celles historiques.

Nous pouvons voir deux raisons principales à ce choix de la Modernité comme sujet privilégié par l'auteure pour son livre : la première, la plus évidente, est certainement le fait que la Modernité soit le système de pensée et de valeurs auquel le régime actuel succède et auquel il se compare. La seconde, moins visible, car non revendiquée, est sans doute que cette période recouvre le chemin parcouru en filigrane par l'histoire pour atteindre une « autonomie épistémologique » (Ricœur, 2000), dans le sillon duquel pourraient se placer les « sciences mémorielles » en développement. Par ailleurs, si ce n'est pas avec la Modernité que le temps est entré comme objet dans l'histoire des idées, c'est à cette époque qu'ont été pensés ensemble temps, mémoire et histoire, et des philosophes tels que Nietzsche, Bergson et Heidegger ont ainsi renouvelé une réflexion existentielle qui sert encore aujourd'hui de cadre d'approche pour ces sujets.

Dans son étude du régime temporel de la Modernité, Aleida Assmann ne fait à aucun moment référence à ces philosophes modernes ou à des contemporains comme Ricœur ${ }^{3}$, leur préférant des références littéraires. Il s'agit là d'un parti pris sur lequel nous pourrons nous interroger, littérature et philosophie ne s'excluant pas l'une l'autre. Son propos débute ainsi par un rappel de la conception du présent chez Baudelaire, pour qui l'homme moderne est représenté par la figure du flâneur, sans mémoire du passé ni attente de l'avenir, livré sans réserve au moment présent. Cette posture reviendrait à nier la conscience de soi telle que définie quelques décennies plus tard par Bergson lorsqu’il écrit que « la mémoire est là, ou bien alors la conscience n'y est pas. [...] Toute conscience est donc mémoire - conservation et accumulation du passé dans le présent. Mais toute conscience est [aussi] anticipation de l'avenir ${ }^{4}$. » Pour débloquer de telles apories, Aleida Assmann cite Shakespeare (le titre de l'ouvrage est inspiré d'une réplique d'Hamlet : « Time is out of joint » ${ }^{5}$ ), Tolstoï ou Virginia Woolf, exemples qui permettent en l'occurrence de dépasser le problème de la conscience de soi en accordant au temps présent une durée, un contenu. Bien que traversée par plusieurs mouvements suivant lesquels la tension entre passé et futur - entre « expérience » et « horizon d'attente » chez Koselleck - a pu varier, l'époque moderne se caractérise par des traits majeurs récurrents. Aleida Assmann en dénombre cinq : le fractionnement du temps (distinction nette entre passé, présent et futur, rupture du nouveau par rapport à l'ancien, instant vécu sans cesse renouvelé et imprévisible, distanciation d'avec la religion et les traditions), l'illusion du commencement (progrès continu, faire table rase du passé pour prendre un nouveau départ), la destruction créatrice (utopies, révolutions, ne pas se retourner et regarder vers l'avenir seulement, capitalisme et production, reconstruction), l'invention de l'historique (historicisme, reconstitution, objectivité, conservation) et l'accélération (vitesse, régularité et précision, rapprochement espace-temps, expérience des changements socioculturels à l'échelle d'une vie, dates 
de péremption, modes). Toutefois, tout comme l'idéal du flâneur, ces concepts se révèlent trop abstraits et intransigeants pour correspondre à l'expérience (ce qui fera dire à Bruno Latour que «nous n’avons jamais été modernes »- les Modernes compris). C'est pourquoi les tenants de la théorie de la « compensation », au premier rang desquels Hermann Lübbe et Odo Marquard, vont rationaliser les concepts des Modernes pour les faire correspondre au rythme anthropologique. Cette « Modernité tardive » affirme notamment la nécessité pour le futur de prendre son origine dans le passé. S’agit-il là d’une première étape vers la fin de la Modernité ou bien ce réajustement a-t-il permis au contraire de faire perdurer la validité des thèses modernistes dans le temps?

Si les Modernes tardifs ont ancré les fondements de la Modernité dans la réalité, force est de constater que cette réalité a changé ces dernières décennies, de telle sorte que certains postulats des Modernes ne semblent plus valides. Il en est ainsi de l'orientation invariable vers le futur d'une part, et de la rupture avec le passé d'autre part. Après les années 1960, qui marquent l'apogée des thèses modernistes, on constate une perte de confiance en le futur, qui n'est plus associé à l'idée de progrès. La frontière avec le passé n'est plus imperméable, ce dernier refaisant surface dans le présent de façon tout à fait inédite après les expériences traumatiques du XXe siècle. Par ailleurs, Aleida Assmann souligne la naissance de trois nouvelles catégories : la culture, l'identité - notamment l'identité collective - et la mémoire, qui forment les piliers des « sciences de la culture » (Kulturwissenschaft) telles qu'elles se sont développées en Allemagne. La mémoire est venue se placer à côté de l'histoire comme moyen d'atteindre un passé qui déborde dans le présent et n'est plus uniquement mis à distance en tant qu'objet d'une science objective. Aleida Assmann prend l'image d'un déplacement de curseur du futur vers le passé. D’autres auteurs comme Andreas Huyssen ( futur présent ») et François Hartog ( trage en même temps qu’un gonflement, un étirement du présent. Un certain nombre d'historiens, anthropologues, politologues perçoivent ainsi la société actuelle comme se dirigeant inéluctablement vers une errance figée dans le présent. Aleida Assmann entre directement en confrontation avec les tenants d'une telle position, qui sont selon elle troublés par cette porosité entre le passé historique et le présent vécu que crée la mémoire collective, en rupture avec les principes de l'historiographie. De fait, les conclusions de la plupart des historiens, en France comme en Allemagne, se rejoignent sur deux points : l'inflation de la notion de mémoire d'une part, qui tend à être privilégiée par rapport à l'histoire, car plus directe, plus accessible et plurielle, donc plus facilement utilisable à des fins identitaires; d'autre part une crainte face au chaos auquel peut mener la coexistence de mémoires multiples et la manipulation subjective du passé qu'elles impliquent, qui brouilleraient ainsi les pistes d'un passé commun et donc d'un avenir commun. L'histoire se méfie ainsi de la mémoire, qu'elle utilise pourtant, semblant oublier qu'elle-même a été et est encore parfois l'objet d'une telle méfiance, car manipulable ou en tout cas pas indépendante des conditions de son écriture, ce qui donna naissance à l'historiographie, à ce regard critique du travail d'historien, de l'histoire sur elle-même. Or au cours de cette « opération 


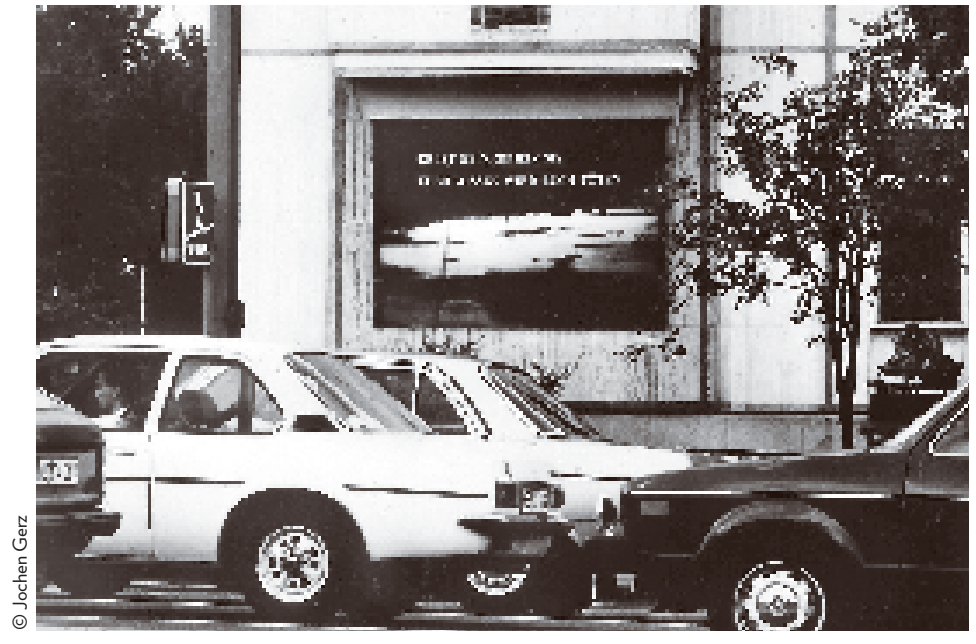

_ Jochen Gerz « Il n'y a pas de mystère C'est votre savoir qui vous tuera ». Paris 1982, Chambéry 1982, Tel Hai 1983. Ici à Kassel, 1984.

(6) Michel de Certeau, Histoire et psychanalyse entre science et fiction, 1987, cité dans François Hartog, Régimes d'historicité. Présentisme et expériences du temps, Paris, Seuil, 2003, p. 11

(7) Andreas Huyssen, «Present Pasts : Media, Politics, Amnesia », in Public Culture, 12/1, Hiver 2000, p. 21-38, ici p. 21, cité p. 14 , traduction de Clotilde Coueille. dans le paysage scientifique. historiographique », Michel de Certeau put observer que « [s] ans doute l'objectivation du passé, depuis trois siècles, avait fait du temps l'impensé d'une discipline qui ne cessait de l'utiliser comme un instrument taxinomique ${ }^{6}$.»

Comment ne pas penser que c'est ce qu'est en train d'éviter Aleida Assmann avec son ouvrage sur notre relation au temps? Autrement dit, l'autonomie épistémologique qu'elle négocie pour la discipline en plein épanouissement dont elle est la chef de file se doterait ici de nouveaux outils de façon aussi légitime que l'histoire. En outre, se basant sur ses propres travaux consacrés à la mémoire collective en Allemagne après la Seconde Guerre mondiale, Aleida Assmann fait le constat que non seulement les études mémorielles ne viennent pas remettre en question la discipline historique, mais qu'au contraire elles se nourrissent de ses acquis afin de répondre au besoin grandissant de la société de s'approprier son passé pour aller de l'avant (p. 279). Il est évident que son but n'est pas de faire peur avec une mémoire toute-puissante, mais d'élever le débat afin que tous les apports disciplinaires puissent être pris en compte. Pour Marc Bloch, l'histoire prend pour objet « les hommes dans le temps ». De la même façon, l'objet des études mémorielles ne pourrait-il pas être défini comme ce «présent des choses passées » pour reprendre le terme de Saint Augustin, ou encore un «passé au présent », qui renverrait à l'« à-présent », terme par lequel Walter Benjamin décrit le processus mémoriel qui rend le passé présent tel qu’on se le remémore, et non forcément tel qu'il était? Les possibilités sont donc nombreuses d'ancrer les études mémorielles dans une pluralité de traditions (philosophique, historique, sociale, politique, littéraire...) qui inscrirait cette pluralité de façon pérenne

C'est dans cet esprit qu'Aleida Assmann rebondit, dans l'introduction, à cette phrase d'Andreas Huyssen : « le focus s'est déplacé d’un futur présent vers des visions présentes du passé et ce changement dans l'expérience et le ressenti du temps nécessite une explication historique et phénoménologique ${ }^{7}$. " L'absence de réponse des sciences de la culture à cette question a en effet motivé l'écriture de son livre. Mais quid de la phénoménologie et de l'héritage heideggerien portant sur le temps et l'historialité? Aleida Assmann fait-elle partie d'une génération en rupture avec cet héritage controversé de la Modernité ? L’auteure devient alors son propre objet d'étude, ou tout au moins le lecteur peut faire cette réflexion et se demander quelle mémoire souhaite Aleida Assmann pour les études mémorielles : quelles traditions de pensée faire revivre et lesquelles oublier pour construire leur identité? 
En France, Claude Romano reprend les apports conceptuels de la phénoménologie et en particulier la terminologie de Heidegger pour les dépasser en introduisant, en miroir du couple conceptuel existentiel/existential, celui d'événementiel/événemential et, partant, une nouvelle approche de l'événement. Or la définition qu'il en donne semble faite pour être appliquée aux drames du XX ${ }^{\mathrm{e}}$ siècle et notamment à la Shoah. Nous pouvons citer ce passage de L'Aventure temporelle, qui laisse entrevoir l'apport potentiel pour les études mémorielles d'une recherche qui prend pleinement possession de son héritage philosophique et permet d'aborder de manière directe et frontale des questionnements étonnamment proches de ceux auxquels tente de répondre le présent ouvrage : «[L]'événement dérange toute chronologie factuelle en même temps que toute coïncidence à soi du présent et toute présence plénière de l'advenant à ce qui lui arrive, il n'est présent que comme passé, à la lumière du futur qu'il ouvre. Il ouvre dans le temps une déhiscence et un hiatus qui en fait un temps disjoint, “hors de ses gonds" selon l'expression de Shakespeare ${ }^{8}$ ».

Se pose dès lors un certain nombre de questions quant à la place des différentes disciplines scientifiques qui étudient le passé aujourd'hui et la relation qu'entretiennent les chercheurs, qu'ils soient historiens ou spécialistes des questions mémorielles, avec la société dans laquelle ils vivent. Notre société est en effet composée dorénavant d'une poignée de professionnels et d'une multitude de mémoires, individuelles et collectives, qui sont le reflet d'un éclatement des conceptions intellectuelles. Sans verser dans un pessimisme qu'Aleida Assmann dénonce, il apparaît donc nécessaire de conserver un esprit critique sur les études mémorielles afin de se prémunir du « tout mémoire », d'une utopie mémorielle, tout comme Nietzsche y enjoignait ses contemporains lorsqu'il dénonçait une dérive du «tout histoire » à l'époque moderne.

Enfin, dans un contexte de globalisation, nous assistons à une harmonisation rapide à l'échelle mondiale de ces mêmes moyens techniques qui avaient créé les conditions de la Modernité en Occident. Cela devrait permettre de poser des repères communs pour l'étude du rapport au temps des différentes cultures, mais faut-il encore que chaque culture s'ouvre à cette internationalisation pour que des mécanismes de transfert soient enclenchés, grâce entre autres à la traduction.

Aleida Assmann a adapté sa démarche à un tel contexte. Bien que conservant un point de vue occidental de par son objet d'étude, le mérite de Ist die Zeit aus den Fugen? est double: il propose à la fois une synthèse de multiples apports théoriques permettant d'appréhender notre propre rapport au temps, en faisant appel à des auteurs d'origines et d'époques différentes, et une complexification de schémas devenus trop simplistes pour cette société plurielle, aux identités fractales et réunies dans un même village mondial. Derrière une démonstration humble et méthodique se dessinerait ainsi un enjeu ambitieux pour les études mémorielles qui prétendraient offrir un espace d'intellection par excellence pour remodeler les cadres conceptuels de la (post)modernité.
(8) Claude Romano, L'Aventure temporelle, Paris, PUF, 2010 p. 79. 